\title{
An overview of algebraic quantum field theory on curved spacetimes
}

\section{Claudio Dappiaggi*}

Dipartimento di Fisica - Università di Pavia

\& INFN, Sezione di Pavia

Via Bassi, 6 - I-27100, Pavia, Italy

E-mail: claudio.dappiaggi@unipv.it

\begin{abstract}
Algebraic quantum field theory is a mathematically rigorous approach to quantum theories which emphasizes, on the one hand, the role and the structural properties of the set of all possible observables and, on the other hand, the choice of a physically acceptable quantum state. We shall review this framework focusing our attention on the simple case of a real scalar field. Our goal will be to highlight the effectiveness of the algebraic approach especially for quantum field theories on curved backgrounds. For this sake we will discuss an application to cosmological models.
\end{abstract}

Proceedings of the Corfu Summer Institute 2015 "School and Workshops on Elementary Particle Physics and Gravity"

1-27 September 2015

Corfu, Greece

${ }^{*}$ Speaker. 
Algebraic quantum field theory is an axiomatic and mathematically rigorous approach to quantum theories which has its origins in the seminal paper of Haag and Kastler [10, 11]. It is based mainly on two key ingredients. The first consists of the assignment to a physical system of a suitable $*$-algebra of observables encoding relevant physical properties such as isotony, locality and covariance, as well as the algebraic relations among observables, e.g., the commutation relations. The second is the identification of an algebraic state, that is a positive and normalized functional over the algebra of observables, out of which one can recover via the celebrated GNS theorem the probabilistic interpretation proper of a quantum theory.

The successes of the algebraic approach are manifold, especially in unveiling several structural properties of quantum field theories. One of the main merits of this viewpoint is its adaptability to be used also on curved backgrounds, regardless of the presence of an underlying non trivial isometry group [8].

Goal of this proceeding is to emphasize this particular last aspect of the algebraic approach making the reader acquainted with the quantization of a Klein-Gordon field on curved backgrounds. Since one of the more common sources of doubts towards algebraic quantum field theory is its degree of abstraction and, thus, its lack of a straightforward connection towards physical models, we will spend some time in discussing a concrete application in a cosmological setting. More precisely we will consider a real, massive and conformally coupled scalar field on a FriedmannRobertson-Walker spacetime with flat spatial sections and we will construct its stress-energy tensor. We will discuss in particular its regularization and the freedoms available in this process, showing that one can use such tensor in a semiclassical version of the Einstein's equations. The resulting dynamical systems turns out to be ruled by an ordinary differential equations admitting two critical points. These can be interpreted as asymptotically stable solutions to the Einstein's equations. As a by-product we will be able to conclude that the Universe is flowing at large times towards a de Sitter phase, hence displaying the existence of an effective cosmological constant whose value cannot be determined by the theory since it depends on suitable regularization parameters.

This proceeding is organized as follows: In Section 1 we introduce the notion of globally hyperbolic spacetimes. These are the manifolds on which the dynamics of free fields can be discussed in terms of an initial value problem. Afterwards we will focus our attention towards a real KleinGordon field and we shall characterize the space of smooth solutions in terms of suitable Green operators. In Section 2, we will develop a notion of classical observable in terms of functionals over the space of dynamically allowed configurations. Starting from these we will be constructing the algebra of fields which encompasses additionally the information about the canonical commutation relations. In Section 3, we will introduce the concept of algebraic state and the so-called Hadamard condition, which allows us to select only those states which are physically meaningful. As an application we will outline the construction of Wick polynomials by using a regularization scheme which relies on Hadamard states. Eventually, in the last section, we shall discuss an application to cosmology and to the semiclassical Einstein's equations in particular.

\section{Geometric and Functional Preliminaries}

As we have anticipated, we will not discuss the algebraic approach to quantum field theory from an axiomatic point of view or in full generality, but via a simple, yet instructive example. 
To this end we need to set a few definitions and conventions, though, in order to keep at bay the length of the presentation, we will focus only on the main ones. Additional details can be found in [4, Chap. 3], on which this section is based. Our starting point is the assignment of a four dimensional, globally hyperbolic spacetime $(M, g)$, that is a connected, smooth, oriented and time oriented manifold $M$ endowed with a Lorentzian metric $g$ of signature $(-,+,+,+)$, such that there exists a Cauchy surface $\Sigma$. This is a closed achronal subset whose domain of definition coincides with $M$ itself.

A useful and more explicit characterization of such class of spacetimes has been obtained by Bernal and Sanchez - see [1, Sect 1.3], who proved that, $(M, g)$ is globally hyperbolic if and only if it is isometric to $\mathbb{R} \times \Sigma$, endowed with the line element

$$
d s^{2}=-\beta d t^{2}+h_{t}
$$

where $t: \mathbb{R} \times \Sigma \rightarrow \mathbb{R}$ is the projection on the first factor, $\beta$ is a smooth, strictly positive, scalar function on $\mathbb{R} \times \Sigma$, while $t \mapsto h_{t}$, is a 1-parameter family of smooth Riemannian metrics. Furthermore, for all $t \in \mathbb{R},\{t\} \times \Sigma$ is a $3 D$, spacelike, smooth Cauchy surface in $M$.

As one can infer from this last characterization, globally hyperbolic spacetimes come together with a distinguished foliation. In other words we have the perfect framework to set up a Cauchy problem for wave-like operators since we have identified both a family of codimension 1 hypersurfaces on which to assign initial data and a time evolution direction. At the same time, the vast majority of the examples of backgrounds considered in physical models falls in this category. We cannot give a complete and exhaustive list, but it is worth mentioning that, besides the obvious case of Minkowski spacetime, also these renown manifolds are globally hyperbolic:

- all Friedmann-Robertson-Walker (FRW) spacetimes, which are $4 D$, homogeneous and isotropic manifolds whose line element reads

$$
d s^{2}=-d t^{2}+a^{2}(t)\left[\frac{d r^{2}}{1-k r^{2}}+r^{2} d \mathbb{S}^{2}(\theta, \varphi)\right]
$$

where $r$ is a strictly positive coordinate, whereas $d \mathbb{S}^{2}(\theta, \varphi)$ is the standard line element of the unit 2-sphere. The unknowns are $a(t)$ a strictly positive, smooth real function and $k$, a parameter, which, up to a normalization can be set to 0,1 or -1 . Depending on such such choice, $\Sigma$ is a constant curvature Riemannian manifold whose model space is a plane, a sphere or an hyperboloid respectively.

- Schwarzschild spacetime, that is a spherically symmetric, stationary solution to the vacuum Einstein equation, whose line element is

$$
d s=-\left(1-\frac{2 M^{\prime}}{r}\right) d t^{2}+\frac{d r^{2}}{1-\frac{2 M^{\prime}}{r}}+r^{2} d \mathbb{S}^{2}(\theta, \varphi),
$$

where $M^{\prime}$ is a parameter which can be interpreted as the mass of the black hole, while $r$ is a coordinate whose domain of definition is $\left(2 M^{\prime}, \infty\right)$.

On top of a globally hyperbolic spacetime $(M, g)$, we can consider any classical free field theory. For definiteness we will restrict our attention to the Klein-Gordon field, which is modelled 
as follows: Let $\phi: M \rightarrow \mathbb{R}$ and let $C^{\infty}(M)$ be the collection of kinematic configurations. Dynamics is ruled by the Klein-Gordon equation

$$
P \phi=\left(\square-\xi R-m^{2}\right) \phi=0,
$$

where $m$ is the mass of the field, $\xi \in \mathbb{R}$ and $R$ is the scalar curvature. If one sets $\xi=0$, then $\phi$ is said to be minimally coupled (to the scalar curvature), whereas, if $\xi=\frac{1}{6}$, we call it conformally coupled. Our ultimate goal is to quantize such dynamical system, but the first step in this direction consists of obtaining a full control of the space of smooth solutions of (1.1). This can be done in a manifestly covariant way since the Klein-Gordon operator $P$ falls is the special case of normally hyperbolic operators. These have been studied thoroughly in the literature and we will recall here the main results adapting them to the case at hand. We shall follow mainly $[1,2]$ and, to start with, we need to identify special classes of functions on $M$ :

- We say that a smooth function $f$ is spacelike compact, $f \in C_{s c}^{\infty}(M)$, if $\operatorname{supp}(f) \cap \Sigma$ is compact for every Cauchy surface $\Sigma \subset M$,

- We say that a smooth function $f$ is timelike compact $f \in C_{t c}^{\infty}(M)$, if $\operatorname{supp}(f) \cap J^{ \pm}(p)$ is compact for every $p \in M$. Here $J^{ \pm}$stands for the causal future (+) and past (-).

The next step consists of introducing the unique advanced (-) and retarded (+) Green operators associated to $P$, that is the maps $G^{ \pm}: C_{t c}^{\infty}(M) \rightarrow C^{\infty}(M)$ such that $P \circ G^{ \pm}=G^{ \pm} \circ P=$ $\left.i d\right|_{C_{t C}^{\infty}(M)}$ and $\operatorname{supp}\left(G^{ \pm}(f)\right) \subseteq J^{ \pm}(\operatorname{supp}(f))$. As a consequence we can introduce the advancedminus-retarded fundamental solution, often also called causal propagator

$$
G \doteq G^{+}-G^{-}: C_{t c}^{\infty}(M) \rightarrow C^{\infty}(M)
$$

such that $P \circ G=G \circ P=0$. In other words $G$ realizes the following isomorphism between topological vector spaces:

$$
\mathscr{S}(M) \doteq\left\{\phi \in C^{\infty}(M) \mid P \phi=0\right\} \simeq \frac{C_{t c}^{\infty}(M)}{P\left[C_{t c}^{\infty}(M)\right]}
$$

If we restrict our attention to any smooth and compactly supported function $f \in C_{0}^{\infty}(M) \subseteq C_{t c}^{\infty}(M)$, it turns out that $G(f) \in C_{s c}^{\infty}(M)$. In other words also the following vector spaces are isomorphic:

$$
\mathscr{S}_{s c}(M) \doteq\left\{\phi \in C_{s c}^{\infty}(M) \mid P \phi=0\right\} \simeq \frac{C_{0}^{\infty}(M)}{P\left[C_{0}^{\infty}(M)\right]} .
$$

\section{The Algebra of Fields}

In the previous section we have shown how to control and to characterize $\mathscr{S}(M)$, the space of dynamically allowed configurations of a real Klein-Gordon field on a globally hyperbolic spacetime. Our next goal is to associate to such system a suitable algebra of observables. There are several possible ways to proceed, ranging from a more axiomatic perspective, as proposed originally in [8], to a more constructive approach, as advocated in [3] or in [4, Chap. 3]. We shall 
follow this last path, starting from the construction of the following class of functionals: For every $f \in C_{0}^{\infty}(M)$, define

$$
F_{f}: \mathscr{S}(M) \rightarrow \mathbb{R}, \quad \phi \mapsto F_{f}(\phi)=\int_{M} d \mu_{g} f(x) \phi(x),
$$

where $d \mu_{g}$ is the metric induced volume measure. Although it is tempting to call these functionals the classical observables associated to a Klein-Gordon field, every reasonable choice for such class should fulfil at least two basic requirements:

1. the set of all observables should be separating the configurations. In our case of interest, this means that, for every $\phi \in \mathscr{S}(M)$, there must exist at least one $f \in C_{0}^{\infty}(M)$ such that $F_{f}(\phi) \neq 0$,

2. the set of all observables should not be redundant. In our case this means that there must not exist $f \in C_{0}^{\infty}(M)$ such that $F_{f}(\phi)=0$ for all $\phi \in \mathscr{S}(M)$.

While the first condition is a by-product of Hahn-Banach theorem since $\mathscr{S}(M) \subset C^{\infty}(M)$, the second one is not met. As a matter of fact, one can realize that, for every $f=P f^{\prime}, f^{\prime} \in C_{0}^{\infty}(M)$, it holds that, for all $\phi \in \mathscr{S}(M)$,

$$
F_{P f^{\prime}}(\phi)=\int_{M} d \mu_{g}\left(P f^{\prime}\right)(x) \phi(x)=\int_{M} d \mu_{g} f^{\prime}(x) P \phi(x)=0,
$$

where, in the second equality, we have integrated by parts, or, equivalently, we have exploited that $P$ is a formally self-adjoint operator with respect to the pairing defined in (2.1). As a consequence of this observation, we have to restrict the class of functionals that we are working with: We consider equivalence classes $[f] \in \frac{C_{0}^{\infty}(M)}{P\left[C_{0}^{\infty}(M)\right]}$ and we define $\mathscr{E}^{o b s}(M)$ as the vector space of classical observables, that is the collection of the following functionals

$$
F_{[f]}: \mathscr{S}(M) \rightarrow \mathbb{R} \quad \phi \mapsto F_{[f]}(\phi) \doteq F_{f}(\phi) .
$$

In view of the previous discussion, the definition of $F_{[f]}$ does not depend on the choice of the representative in the equivalence class and, for all practical purposes, we can always identify $\mathscr{E}^{\circ b s}(M)$ with the labelling space $\frac{C_{0}^{\infty}(M)}{P\left[C_{0}^{\infty}(M)\right]}$. The set of classical observables is a symplectic space if endowed with the following weakly non-degenerate, antisymmetric, bilinear form:

$$
\sigma: \mathscr{E}^{o b s}(M) \times \mathscr{E}^{o b s}(M) \rightarrow \mathbb{R}, \quad\left(F_{[f]}, F_{\left[f^{\prime}\right]}\right) \mapsto \sigma\left(F_{[f]}, F_{\left[f^{\prime}\right]}\right) \doteq \int_{M} d \mu_{g} f(x) G\left(f^{\prime}\right)(x),
$$

where $G$ is the causal propagator (1.2). The pair $\left(\mathscr{E}^{\circ o b}(M), \sigma\right)$ is the building block to associate to a Klein-Gordon field a full-fledged (quantum) algebra of observables. As a matter of fact $\mathscr{E}$ obs $(M)$ includes already the information on the dynamics, but it does not allow us to discuss the simultaneous measurement of different observables, nor it does account for the canonical commutation relations. These two shortcomings can be overcome as follows: We start from $\mathscr{E}^{\circ o b s}(M ; \mathbb{C}) \doteq \mathscr{E}^{\circ o b s}(M) \otimes \mathbb{C}$ and we construct the universal tensor algebra

$$
\mathscr{T}_{\mathscr{E}}(M) \doteq \bigoplus_{n=0}^{\infty} \mathscr{E}^{o o b s}(M ; \mathbb{C})^{\otimes n}, \quad \mathscr{E}^{o b s}(M ; \mathbb{C})^{\otimes 0}=\mathbb{C} .
$$


Within $\mathscr{T}_{\mathscr{E}}(M)$, we can identify a two-sided ideal $\mathscr{I}_{\mathscr{E}}(M)$ which is generated by elements of the form $F_{[f]} \otimes F_{\left[f^{\prime}\right]}-F_{\left[f^{\prime}\right]} \otimes F_{[f]}-i \sigma\left(F_{[f]}, F_{\left[f^{\prime}\right]}\right) \mathbb{I}$, where $\mathbb{I}$ is the identity element of $\mathscr{T}_{\mathscr{E}}(M)$ and $\sigma$ is the symplectic form (2.2). In order to implement the canonical commutation relations it suffices to consider the following quotient of algebras:

$$
\mathscr{F}(M)=\frac{\mathscr{T}_{\mathscr{E}}(M)}{\mathscr{I}_{\mathscr{E}}(M)},
$$

This is the so-called algebra of fields and it encodes the observables of the quantum theory of a Klein-Gordon field. Furthermore it enjoys the following notable properties:

1. $\mathscr{F}(M)$ is a unital $*$-algebra, with respect to the $*$-operation inherited from complex conjugation over $\mathscr{E}^{o b s}(M ; \mathbb{C})$,

2. it is causal, namely every pair of elements $\Phi, \Phi^{\prime} \in \mathscr{F}(M)$, which are causally disjoint, commute.

3. it fulfils the time-slice axiom: For every globally hyperbolic open neighbourhood $\mathscr{O}$ of a spacelike Cauchy surface, there exists a $*$-isomorphism between $\mathscr{F}(M)$ and $\mathscr{F}(\mathscr{O})$, the algebra of fields built over $\left(\mathscr{O},\left.g\right|_{\mathscr{O}}\right)$.

\section{Hadamard States and Wick Polynomials}

In the previous section we have outlined a constructive scheme to assign an algebra of observables to a free field theory, such as the Klein-Gordon field. Nonetheless the quantization process is far from being complete since we have not recovered yet the probabilistic interpretation proper of all quantum theories. This can be obtained assigning one last ingredient, namely an algebraic state (for short, simply a state), that is a linear functional

$$
\omega: \mathscr{F}(M) \rightarrow \mathbb{C}, \quad \omega(\mathbb{I})=1, \quad \omega\left(a^{*} a\right) \geq 0 \quad \forall a \in \mathscr{F}(M),
$$

where $\mathbb{I}$ is the identity of the algebra of fields. Notice that the second condition, also known as positivity, is a highly non linear constraint; hence, constructing a state is not a straightforward operation. The advantage of considering a pair such as $(\mathscr{F}(M), \omega)$ lies in the renown GNS theorem, see for example [4, Chap. 5], which tells us that we can associate to $(\mathscr{F}(M), \omega)$ a triple $\left(\mathscr{D}_{\omega}, \pi_{\omega}, \Omega_{\omega}\right)$. Here $\mathscr{D}_{\omega}$ is a dense subspace of a Hilbert space $\mathscr{H}_{\omega}$, while $\pi_{\omega}: \mathscr{F}(M) \rightarrow \mathscr{L}\left(\mathscr{D}_{\omega}\right)$ is $*$-representation. Furthermore $\Omega_{\omega}$ is a unit norm element of $\mathscr{D}_{\omega}$ such that $\mathscr{H}_{\omega}=\overline{\pi_{\omega}(\mathscr{F}(M)) \Omega_{\omega}}$. The so-called GNS triple is unique up to unitary equivalence.

We observe that, in view of the isomorphism between $\mathscr{E}^{\circ o b s}(M ; \mathbb{C})$ and $\frac{C_{0}^{\infty}(M ; \mathbb{C})}{P\left[C_{0}^{\infty}(M ; \mathbb{C})\right]}$, one way to define a state on $\mathscr{F}(M)$ consists of assigning $n$-point correlation functions. These are continuous linear maps

$$
\omega_{n}: \underbrace{C_{0}^{\infty}(M ; \mathbb{C}) \times \ldots \times C_{0}^{\infty}(M ; \mathbb{C})}_{n} \rightarrow \mathbb{C},
$$

that is elements of $D^{\prime}(M \times \ldots \times M)$ which are weak solutions to the Klein-Gordon equation (1.1) subject to additional constraints needed to ensure compatibility both with the canonical commutation relations defining $\mathscr{I}_{\mathscr{E}}(M)$ and with positivity as per (3.1). A simpler version of this procedure 
arises from the additional assumption that all odd correlation functions are vanishing, $\omega_{2 n+1}=0$ for all $n \in \mathbb{N}$, while the even ones are completely determined by the two-point function via the following defining formula:

$$
\omega_{2 n}\left(f_{1} \otimes \ldots \otimes f_{2 n}\right)=\sum_{\pi_{2 n} \in S_{2 n}} \prod_{i=1}^{n} \omega_{2}\left(f_{\pi_{2 n}(2 i-1)} \otimes f_{\pi_{2 n}(2 i)}\right),
$$

where $S_{2 n}$ is the group of ordered permutations of $2 n$ elements, while $f_{i} \in C_{0}^{\infty}(M ; \mathbb{C})$ for all $i=$ $1, \ldots, n$. In order to use (3.2) to construct a full-fledged algebraic state, a necessary condition is the identification of a bi-distribution $\omega_{2} \in \mathscr{D}^{\prime}(M \times M)$ such that, for all $f, f^{\prime} \in C_{0}^{\infty}(M ; \mathbb{C})$

$$
\omega_{2}\left(P f, f^{\prime}\right)=\omega_{2}\left(f, P f^{\prime}\right)=0, \quad \omega_{2}(\bar{f}, f) \geq 0 \quad \text { and } \quad \omega_{2}\left(f, f^{\prime}\right)-\omega_{2}\left(f^{\prime}, f\right)=i \sigma\left(F_{[f]}, F_{\left[f^{\prime}\right]}\right),
$$

where $P$ is the Klein-Gordon operator (1.1), while $\sigma$ is the symplectic form (2.2). A state on $\mathscr{F}(M)$ built out of a two-point function, subject to these conditions, is known as a quasi-free/Gaussian state. From a physical point of view, such class of states is noteworthy since the Hilbert space of the associated GNS triple is always of Fock type, an assumption common in all key applications.

Even restricting our attention to Gaussian states, it is hardly conceivable that all of them can be considered physically acceptable and a selection criterion needs to be formulated. Such problem has been thoroughly studied in the past decades culminating with the seminal work of Radzikowski $[18,19]$. From a physical perspective a set of natural requirements to be imposed to an algebraic state are the following: its ultraviolet behaviour coincides with that of the Minkowski vacuum and all quantum fluctuations of the observables are finite. At a mathematical level this translates in the celebrated Hadamard condition, which, for Gaussian states, is a constraint on the singular structure of the two-point function $\omega_{2}$. Although the best way to present it relies on the notion of wavefront set, a tool proper of microlocal analysis [4, Chap. 5], we would need to introduce additional mathematical structures, not fitting in this review. An alternative and, to some extent, more informative way of presenting the Hadamard condition is to discuss it at a local level. In other words, we say that $\omega_{2} \in \mathscr{D}^{\prime}(M \times M)$ is (locally) of Hadamard form if, for every pair of points $x, y \in M$ lying in a geodesic neighbourhood, the integral kernel of the bi-distribution reads

$$
\begin{gathered}
\omega_{2}(x, y)=\lim _{\varepsilon \rightarrow 0} \frac{1}{8 \pi^{2}}\left(\frac{u(x, y)}{\sigma_{\varepsilon}(x, y)}+v(x, y) \log \left(\frac{\sigma_{\varepsilon}(x, y)}{\lambda^{2}}\right)+w(x, y)\right) \\
\doteq \lim _{\varepsilon \rightarrow 0} \frac{1}{8 \pi^{2}}\left(h_{\varepsilon}(x, y)+w(x, y)\right) .
\end{gathered}
$$

Here $\lambda$ is an arbitrary scale length, while

$$
\sigma_{\varepsilon}(x, y) \doteq \sigma(x, y)+2 i \varepsilon(t(x)-t(y))+\varepsilon^{2} \quad \varepsilon \in \mathbb{R},
$$

where $t$ is a time function on $(M, g)$ and $\sigma(x, y)$ is the half, squared geodesic distance between $x$ and $y$. The remaining unknowns in (3.3) are the Hadamard coefficients $u, v$, and $w$. These are smooth, real-valued functions, where both $u$ and $v$ depend only on the background metric and on the operator ruling the dynamics, (1.1) in our case. On the contrary $w$ has no constraint barring the one coming from the requirement that $\omega_{2}$ solves the Klein-Gordon equation in a weak sense. The bidistribution $h_{\varepsilon}$ is called Hadamard parametrix and it includes all singular terms in the two-point function. 
The existence of Hadamard states has been established already at the beginning of the eighties thanks to a deformation argument [9], but the construction of explicit examples has been a daunting task, which was at the hearth of many works in the past decade. The reason for such interest towards Hadamard states stems from their pivotal role in the construction of Wick polynomials on a generic globally hyperbolic spacetimes. Such problem has been thoroughly investigated by Hollands and Wald [13] and the starting point is the observation that a quantity such as $\omega\left(\phi^{2}(f)\right)$, the expectation value of a squared scalar field, cannot be defined meaningfully since it would require to test the bi-distribution $\omega_{2}(x, y)$ against $f(x) \delta(x, y), f \in C_{0}^{\infty}(M)$, an ill-defined operation. Exactly as in the standard approach to quantum field theory on Minkowski spacetime, the way out from this quandary consists of regularizing suitably all these ill-defined quantities. If we consider only Hadamard states as those physically acceptable, it is possible to introduce a covariant regularization scheme which does not depend on the choice of the state and which coincides with the usual normal ordering procedure on Minkowski spacetime. This possibility relies strongly on (3.3) and, more precisely, on the dependence of the singular terms only on the geometry of the background and on (1.1). More precisely we set

$$
\omega\left(: \widehat{\Phi}^{2}(x):\right) \doteq \lim _{x \rightarrow y}\left(\omega_{2}(x, y)-\frac{h_{\varepsilon}(x, y)}{8 \pi^{2}}\right),
$$

where $h_{\mathcal{E}}(x, y)$ is the Hadamard parametrix. Wick polynomials are the building block for the treatment of interacting theories at a perturbative level. Starting from (3.4), we can define products such as : $\widehat{\Phi}^{2}(x):: \widehat{\Phi}^{2}(y)$ : by means of Wick's theorem and we can also discuss the regularization of (products of) derivatives of fields following the same procedure outlined in [13, 14].

We have discussed the basic ingredients of the algebraic quantization of a real, massive scalar field on a globally hyperbolic spacetime. Applications of this approach are manifold and they are mainly aimed at understanding better the structural and mathematical properties of quantum field theory. Nonetheless the algebraic approach is suited also for answering more practical questions and for being used in concrete models. In the next section we shall give an example supporting this statement.

\section{A Cosmological Application}

Henceforth we consider a real, massive and conformally coupled scalar field (i.e., $\xi=\frac{1}{6}$ in (1.1)) on a Friedmann-Robertson-Walker (FRW) spacetime with flat spatial sections. In other words $M \simeq I \times \mathbb{R}^{3}$, where $I \subseteq \mathbb{R}$ and, taking the standard Cartesian coordinates, the line element reads $d s^{2}=-d t^{2}+a^{2}(t)\left[d x^{2}+d y^{2}+d z^{2}\right]$. Here $a$ is a smooth and strictly positive function which has to be determined solving the Einstein's equations. In this context, one might wonder what happens if, in place of a classical stress-energy tensor, we consider on the right hand side of these equations its quantum counterpart. In other words we are switching to a semiclassical regime whose dynamics is ruled by the so-called semiclassical Einstein's equations:

$$
G_{\mu v}=8 \pi \omega\left(: T_{\mu \nu}:\right),
$$

where $G_{\mu v}$ is the Einstein tensor, while : $T_{\mu v}$ : is the Wick-ordered stress-energy tensor. Being, per construction, an approximated version of a full quantum theory, one might wonder whether 
these equations are mathematically meaningful and, in which regime, they can be used reliably. These questions have been thoroughly investigated since the end of the seventies [20,21] and also recently in the context of homogeneous and isotropic spacetimes - see [16, 17] and [5], on which our analysis is based.

The classical stress energy tensor of a real, massive, conformally coupled scalar field $\phi$ is

$$
T_{\mu v}[\phi]=\partial_{\mu} \phi \partial_{\nu} \phi-\frac{1}{6} g_{\mu \nu}\left(\partial_{\rho} \phi \partial^{\rho} \phi+m^{2} \phi^{2}\right)-\frac{1}{6} \nabla_{\mu} \partial_{\nu} \phi^{2}+\frac{1}{6}\left(R_{\mu v}-\frac{R}{6} g_{\mu \nu}\right) \phi^{2},
$$

where $R_{\mu v}$ and $R$ are the Ricci tensor and the scalar curvature built out of the metric. We observe that, for all $\phi \in \mathscr{S}(M), \nabla^{\mu} T_{\mu v}[\phi]=0$ and $T[\phi] \doteq g^{\mu v} T_{\mu v}[\phi]=-m^{2} \phi^{2}$. The first identity is especially relevant since it expresses the compatibility of the stress-energy tensor with the Eisntein tensor, which is in turn divergence free. We remark additionally that (4.1) differs from the expression of $T_{\mu \nu}[\phi]$ more commonly found in the literature for the following term:

$$
\frac{1}{3} g_{\mu v}(\phi P \phi+(P \phi) \phi)
$$

where $P$ is the operator in (1.1) with $\xi=\frac{1}{6}$. At a classical level this term does not contribute whenever $\phi \in \mathscr{S}(M)$, but it plays a key role at a quantum level.

As a matter of fact, if we start from the Lagrangian density $\mathscr{L}[\phi]$ for a Klein-Gordon, conformally coupled scalar field and we define the stress-energy tensor as usual as $T_{\mu \nu}^{\mathscr{L}}[\phi]=-\frac{2}{\sqrt{|g|}} \frac{\delta \mathscr{L}[\phi]}{\delta g^{\mu \nu}}$, it turns out that, after the Wick regularization, the resulting $: T_{\mu \nu}^{\mathscr{L}}:$ is not divergence free. Hence it is not compatible with the Einstein tensor, making the semiclassical Einstein's equations ill-defined. A way out, studied in [14], consists of noticing that there is a certain leeway in the definition of the stress-energy tensor, which consists of adding terms which, at a classical level, are both divergence free and traceless if $\phi \in \mathscr{S}(M)$. Yet, there exists only one such term, namely (4.2), which, after the Wick regularization, yields a full stress-energy tensor which is divergence free. The price to pay for this alternative choice is a modification at a quantum level of the expectation value of the regularized trace of $T_{\mu \nu}$, namely

$$
\omega(: T:)=-\frac{m^{2}}{8 \pi^{2}} \omega\left(: \phi^{2}:\right)+\frac{1}{2880 \pi^{2}}\left(C_{\mu v \rho \delta} C^{\mu v \rho \delta}+R_{\mu v} R^{\mu \nu}-\frac{R^{2}}{3}+\square R\right)+\frac{m^{4}}{32 \pi^{2}},
$$

where $C_{\mu v \rho \delta}$ is the Weyl tensor. The extra term in (4.3) is usually called the trace anomaly. Our attention towards the expectation value of the regularized trace is motivated especially by the presence of only one degree of freedom in the FRW metric, the scale factor $a(t)$. To determine it, it is not necessary to use the whole semiclassical Einstein's equations, but it suffices to consider the trace:

$$
-R=8 \pi^{2} \omega(: T:) .
$$

If we were to insert (4.3) in this equation, we would be dealing in general with a differential equation depending on the fourth derivative of the metric coefficients on account of the term proportional to $\square$. Hence we would be expecting to find unstable solutions, a rather disappointing feature for a physical model. Nonetheless a way out from this setback stems from the realization [21] that the 
Wick regularization based on (3.4) is not uniquely defined and we have still a geometric freedom, which, in the case of the stress energy tensor, translates to

$$
: T_{\mu \nu}: \rightarrow: T_{\mu \nu}:+A I_{\mu \nu}+B J_{\mu \nu}
$$

where $A, B$ are arbitrary real constants while

$$
I_{\mu v}=\frac{\delta}{\delta g^{\mu \nu}} \int_{M} d \mu_{g} R^{2}, \quad J_{\mu v}=\frac{\delta}{\delta g^{\mu v}} \int_{M} d \mu_{g} R_{\rho \delta} R^{\rho \delta} .
$$

At a level of trace both $I_{\mu \nu}$ and $J_{\mu \nu}$ account for a term proportional to $\square R$ and hence, our regularization freedom amounts to

$$
: T: \mapsto: T:+C \square R,
$$

where $C$ is an arbitrary real constant. We can exploit such freedom selecting the value of $C$ in such a way that the term proportional to $\square R$ in (4.3) disappears. If we recall that all spacetimes, whose metric is conformal to the Minkowski one, have a vanishing Weyl tensor, (4.4) becomes an ordinary differential equation:

$$
-6\left(\dot{H}+2 H^{2}\right)=-8 \pi m^{2} \omega\left(: \phi^{2}:\right)+\frac{1}{\pi}\left(-\frac{1}{30}\left(\dot{H} H^{2}+H^{4}\right)+\frac{m^{4}}{4}\right),
$$

where $H=\frac{\dot{a}}{a}$ and the dot stands for the time derivative. Before discussing the solutions to (4.5), we need to spend a few words on the choice of the state $\omega$. Having used the regularization based on (3.4), we have already assumed implicitly that $\omega$ is of Hadamard form. For consistency with the form of (4.5), we need also that $\omega$, or equivalently its two-point function $\omega_{2}$ is invariant under the action of all background isometries, namely the three-dimensional Euclidean group, for a FRW metric with flat spatial sections. The existence and the explicit construction of such kind of homogeneous and isotropic Hadamard states has been thoroughly investigated in the past years, see $[6,7,15]$.

As discussed in [5], for our purposes we consider those Hadamard states such that $\omega\left(: \phi^{2}:\right)=$ $\alpha m^{2}+\beta R$, where $\alpha$ and $\beta$ are arbitrary real numbers. This transforms (4.5) in the following ODE:

$$
\dot{H}\left(H^{2}-H_{0}^{2}\right)=-H^{4}+2 H_{0}^{2} H^{2}+M,
$$

where

$$
H_{0}^{2}=180 \pi\left(1-8 \pi m^{2} \beta\right), \quad M=\frac{15}{2} m^{4}-240 \pi^{2} m^{4} \alpha .
$$

We observe that (4.6) has two critical points namely $H_{ \pm}^{2}=H_{0}^{2} \pm \sqrt{H_{0}^{4}+M}$, which can be set to be positive or, at most one, equal to zero by fixing suitably the values of $\alpha$ and $\beta$. A qualitative analysis of (4.6) shows that both $H_{+}$and $H_{-}$are asymptotically stable solutions, whose physical interpretation is the following: The semiclassical Einstein's equations yield solutions which are flowing at large time to a de Sitter phase. This translates into the existence of an effective cosmological constant whose value cannot be fixed a priori since it depends on a regularization freedom.

This result proves that the algebraic approach to quantum field theory on curved backgrounds can be used effectively also to discuss concrete physical models and the application that we have shown can be thought as a first attempt in this direction. A reader, who is interested in further developments along these lines, is strongly encouraged to refer to [12]. 


\section{Acknowledgments}

The author is grateful to the organizers of the School and Workshops on Elementary Particle Physics and Gravity, as well as to the Humboldt Foundation, for the kind invitation and for the financial support. The work of C.D. is supported also by the University of Pavia.

\section{References}

[1] C. Bär, Green-hyperbolic operators on globally hyperbolic spacetimes, Comm. Math. Phys. 333 (2015), no. 3, 1585-1615.

[2] C. Bär, N. Ginoux and F. Pfaffle, Wave equations on Lorenzian manifolds and quantization, Eur. Math. Soc., Zürich, 2007.

[3] M. Benini, C. Dappiaggi and T. P. Hack, Quantum Field Theory on Curved Backgrounds - A Primer, Int. J. Mod. Phys. A 28 (2013) 1330023, [arXiv:1306.0527 [gr-qC]].

[4] R. Brunetti, C. Dappiaggi, K. Fredenhagen and J. Yngvason, Advances in algebraic quantum field theory, Springer, 2015.

[5] C. Dappiaggi, K. Fredenhagen and N. Pinamonti, Stable cosmological models driven by a free quantum scalar field, Phys. Rev. D 77 (2008) 104015, [arXiv:0801.2850 [gr-qc] ].

[6] C. Dappiaggi, V. Moretti and N. Pinamonti, Cosmological horizons and reconstruction of quantum field theories, Commun. Math. Phys. 285 (2009) 1129, [arXiv:0712.1770 [gr-qc] ].

[7] C. Dappiaggi, V. Moretti and N. Pinamonti, Distinguished quantum states in a class of cosmological spacetimes and their Hadamard property, J. Math. Phys. 50 (2009) 062304, [arXiv: 0812.4033 $[\mathrm{gr}-\mathrm{qC}]]$.

[8] J. Dimock, Algebras of Local Observables on a Manifold, Commun. Math. Phys. 77, 219 (1980).

[9] S. A. Fulling, F. J. Narcowich and R. M. Wald, Singularity Structure Of The Two Point Function In Quantum Field Theory In Curved Space-Time, I', Ann. Phys. 136, 243 (1981).

[10] R. Haag, Local Quantum Physics: Fields, Particles, Algebras, 1st edn., Springer, Berlin, 1992.

[11] R. Haag and D. Kastler, An Algebraic approach to quantum field theory, J. Math. Phys. 5, 848 (1964).

[12] T. P. Hack, Cosmological Applications of Algebraic Quantum Field Theory in Curved Spacetimes, SpringerBriefs in Mathematical Physics, Springer, 2015, arXiv:1506.01869 [gr-qc].

[13] S. Hollands and R. M. Wald, Local Wick polynomials and time ordered products of quantum fields in curved space-time, Commun. Math. Phys. 223 (2001) 289, [gr-qc/0103074].

[14] V. Moretti, Comments on the stress energy tensor operator in curved space-time, Commun. Math. Phys. 232, 189 (2003), arXiv: gr-qc/0109048.

[15] H. Olbermann, States of low energy on Robertson-Walker spacetimes, Class. Quant. Grav. 24,5011 (2007), arXiv:0704.2986 [gr-qC].

[16] N. Pinamonti, On the initial conditions and solutions of the semiclassical Einstein equations in a cosmological scenario, Commun. Math. Phys. 305 (2011) 563 [arXiv:1001.0864 [gr-qc]].

[17] N. Pinamonti and D. Siemssen, Global Existence of Solutions of the Semiclassical Einstein Equation for Cosmological Spacetimes, Commun. Math. Phys. 334 (2015) no.1, 171 [arXiv: 1309.6303 [math-ph] ]. 
[18] M. J. Radzikowski, Micro-local approach to the Hadamard condition in quantum field theory on curved space-time, Commun. Math. Phys. 179 (1996) 529.

[19] M. J. Radzikowski, A Local to global singularity theorem for quantum field theory on curved space-time, Commun. Math. Phys. 180 (1996) 1.

[20] R. M. Wald, The Back Reaction Effect in Particle Creation in Curved Space-Time, Commun. Math. Phys. 54 (1977) 1.

[21] R. M. Wald, Trace Anomaly of a Conformally Invariant Quantum Field in Curved Space-Time, Phys. Rev. D 17 (1978) 1477. 